\title{
Severe acute respiratory syndrome (SARS) in neonates and children
}

\section{A M Li, P C Ng}

Arch Dis Child Fetal Neonatal Ed 2005;90:F461-F465. doi: 10.1136/adc.2005.075309

Severe acute respiratory syndrome (SARS) runs a more benign course in children during the acute phase. Infants born to mothers with the disease did not acquire the infection through vertical transmission. The treatment strategy for children with SARS has not been standardised and is based on adult experience. Thus far, no deaths have been reported in the paediatric age group. Exercise impairment and residual radiological abnormalities were present six months after diagnosis. It is important to assess these patients on a regular basis to detect and provide appropriate management for any persistent or emerging long term sequelae in the physical, psychological, and social domains. This review describes the current understanding of SARS coronavirus infection in newborns and children.

See end of article for authors' affiliations $\ldots \ldots \ldots \ldots \ldots \ldots \ldots \ldots$

Correspondence to: Professor $\mathrm{Ng}$, Department of Paediatrics, Level 6 , Clinical Sciences Building, Prince of Wales Hospital, Shatin, NT, Hong Kong; pakcheungng@cuhk.edu.

Accepted 24 May 2005
A lthough severe acute respiratory syndrome (SARS) has wreaked havoc in South East Asia and other parts of the world, it appears to be a disease that predominantly affects adults. Less than 10\% of those infected were children. Among the affected children, only $5 \%$ required admission to an intensive care unit, and less than 1\% required mechanical ventilation. No deaths were reported among the children affected by SARS. In contrast with its adult counterpart, the clinical course of affected children is usually milder, time to resolution is shorter, and the potential of children to infect others is low. These very different features in children have led some to believe that SARS is a relatively mild disease in this age group. The following review will provide an up to date account of this novel disease in neonates and children.

\section{EPIDEMIOLOGY OF SARS}

SARS was brought to Hong Kong in February 2003 by an infected medical doctor, the index case of this epidemic. He arrived from Guangdong province, which is situated about $100 \mathrm{~km}$ north of Hong Kong, and he stayed in a local hotel. It has been estimated that at least 12 guests/visitors in this hotel became infected through contact with this medical doctor directly or indirectly. The disease then spread from Hong Kong rapidly to Hanoi, Singapore, and Toronto when infected guests returned to their home countries. One of the infected visitors from the hotel was admitted to the Prince of Wales
Hospital in early March with pneumonia, and he subsequently infected 138 hospital staff, patients, and visitors. ${ }^{1}$ The use of nebulised medications which generated a large amount of infective droplets was believed to have caused this extensive nosocomial spread of disease. The first few paediatric cases were household contacts of the initial cohort of adult patients from the hospital outbreak. The disease involving healthcare workers then spread rapidly to the community by visitors to the medical wards. The primary mode of transmission appears to be mediated through direct mucous membrane (eyes, nose, and mouth) contact with droplets and/or fomites. ${ }^{12}$

Most if not all children with SARS have either been in close contact with infected adults, as a household contact or in a healthcare setting. ${ }^{3}$ This was believed to be the important route of transmission that put children at a particular risk. Surprisingly, in Hong Kong there had been no major spread of the disease among classmates in schools. This could be explained by the early strict hygiene precautions undertaken by schools following a large scale educational and preventive programme conducted by the local government.

\section{VIROLOGY OF SARS}

SARS is now known to be caused by a novel coronavirus (SARS-CoV), and over $95 \%$ of well characterised cohorts of patients had evidence of recent SARS-CoV infection. ${ }^{12-8}$ Coinfection with human metapneumovirus or other pathogens has also been documented in a proportion of patients. Whether such coinfections contributed to enhancing the pathogenesis or transmission of the disease remains unanswered. ${ }^{9}{ }^{10}$

The genome of SARS-CoV indicates that it is a novel virus within the family of coronaviridae, a group of enveloped positive sense RNA viruses. ${ }^{11}$ It is not related to any of the human or animal coronaviruses known to date. Viruses closely related to SARS-CoV have recently been isolated from animals such as civet cats. ${ }^{12}$ It is postulated that SARS-CoV was an animal virus that had overcome the species barrier and adapted to human to human transmission. The presence of this animal reservoir may imply possible future animal to human transmission and the initiation of further disease outbreaks.

Abbreviations: CoV, coronavirus; $\mathrm{HRCT}$, high resolution computed tomography; RT-PCR, reverse transcriptasepolymerase chain reaction; SARS, severe acute respiratory syndrome 
Table 1 Presenting clinical features $(\%)$ in paediatric series of severe acute respiratory syndrome

\begin{tabular}{|c|c|c|c|}
\hline & $\begin{array}{l}\text { Hon ef } a l^{16} \\
(n=10)\end{array}$ & $\begin{array}{l}\text { Chiv et al }{ }^{8} \\
(n=21)\end{array}$ & $\begin{array}{l}\text { Bitnun et } a l^{17} \\
(\mathrm{n}=10)\end{array}$ \\
\hline Fever & 100 & 91 & 100 \\
\hline Malaise & 20 & 62 & 10 \\
\hline Chills or rigor & 50 & 48 & 10 \\
\hline Myalgia & 40 & 10 & - \\
\hline Cough & 80 & 43 & 60 \\
\hline Dyspnoea & - & 14 & 10 \\
\hline Headache & 40 & 14 & 10 \\
\hline Dizziness & 10 & 38 & - \\
\hline Sputum production & - & 14 & - \\
\hline Sore throat & 30 & 5 & 10 \\
\hline Coryza & 60 & 33 & 40 \\
\hline Anorexia & - & 57 & - \\
\hline Nausea/vomiting & 20 & - & 20 \\
\hline Diarrhoea & - & 10 & 10 \\
\hline Chest pain & - & - & - \\
\hline Abdominal pain & 10 & - & - \\
\hline Febrile convulsion & 10 & - & - \\
\hline Rash & - & 5 & - \\
\hline
\end{tabular}

\section{NEWBORNS OF MOTHERS WITH SARS}

During the outbreak in Hong Kong, 12 pregnant women were diagnosed to have the disease. ${ }^{13}$ Seven mothers presented in the first trimester between three and 12 weeks of gestation, and the rest were in their late second and third trimester. Apart from one case, all pregnant women received ribavirin and systemic corticosteroids. Six (50\%) were admitted to the intensive care unit because of hypoxaemia. Four (33\%) required mechanical ventilation, three of whom $(25 \%)$ died from respiratory failure or nosocomial infection. ${ }^{13}$

Four of the seven pregnant women presenting in the first trimester had spontaneous miscarriages, and two opted for termination of pregnancies because of social reasons. The only newborn survivor in this group was born to the mother who had mild disease and did not receive antiviral or corticosteroid treatment. This infant was delivered at term, and the birth weight was appropriate for gestation. All five newborns in the second and third trimester group survived. ${ }^{14}$ Three were delivered during the acute phase of the illness at 26-32 weeks gestation, and the others in the convalescent phase at 33 weeks gestation and at term.

None of the newborns from SARS affected mothers had clinical, laboratory, or radiological evidence suggestive of SARS-CoV infection. A thorough search for the coronavirus, including serial reverse transcriptase-polymerase chain reaction (RT-PCR) and viral cultures of blood, cerebrospinal fluid, respiratory secretions, excreta, and other body fluids, found no presence of the virus, and paired acute and convalescent sera also did not show a significant (fourfold) increase in titres. ${ }^{14}$ The five newborn infants whose mothers received ribavirin, a potentially teratogenic agent, did not develop any major congenital malformations. ${ }^{14}{ }^{15}$ An important observation from our cohort of newborns of SARS affected mothers is that the three infants delivered soon after the mothers presented with respiratory symptoms had appropriate birth weight for gestation, whereas the infants from the two pregnancies that did not require early obstetric intervention developed oligohydramnios and severe intrauterine growth retardation. ${ }^{13}$ These complications could be related to prolonged usage of high dose systemic corticosteroids or antiviral agents and/or the impact of a severe maternal debilitating illness on normal fetal growth. ${ }^{13}{ }^{14}$ It was, however, unlikely that the manifestations were secondary to transplacental SARS-CoV infection, as no virus or viral particles were demonstrated in the products of conception. ${ }^{14}$ The severe gastrointestinal complications, necrotising enterocolitis and jejunal perforation, observed in the preterm infants are worrying. ${ }^{14}$ Although these findings may have been coincidental, it could be associated with maternal hypoxic-ischaemic insult and/or the use of high dose antenatal corticosteroids, which may have crossed the placenta, or postnatal prostaglandin modulating drugs such as indomethacin, which caused disruption of the gastrointestinal mucosal integrity.

\section{CLINICAL PRESENTATION OF SARS IN CHILDREN AND ADOLESCENTS}

The incubation period of SARS was 2-10 days, and the mean has been estimated to be 6.4 days (95\% confidence interval 5.2 to 7.7$)$. The mean time from onset of clinical symptoms to hospital admission was 3-5 days (e-SARS database, Hospital Authority, HKSAR, data on file). The ratio of infected young children $(<12$ years of age) to adolescents $(12-18$ years of age) was 1:2. Both sexes were equally affected. The overall attack rate was estimated to be 8.9 cases per 100000 children $(<18$ years). Table 1 summarises the frequency of common presenting symptoms from several paediatric series. ${ }^{16-18}$ The predominant and most consistent symptom was fever, which was present in most of the patients $(>90 \%)$ diagnosed to have SARS. Other symptoms include coryza and cough. Chills, rigor, myalgia, and malaise, which are common in adult patients, were also present in older children and adolescents, but were rare in young children. Some patients, adults and children alike, presented with diarrhoea. Young children appeared to have milder disease with a shorter time to resolution, whereas the course of disease in older children resembled that of adults. Physical examination at presentation was normal in almost all young children, whereas inspiratory crepitations over the lung bases were present in some adolescent and adult patients.

The typical clinical course of adult patients with SARS was described as following a triphasic pattern. ${ }^{7}{ }^{19}$ Phase 1 was the viral replication phase characterised by fever, myalgia, and other constitutional symptoms. This phase was associated with an increase in body viral load..$^{7}$ It was transient and the symptoms were expected to improve after a few days. Phase 2 was the immunopathological phase and characterised by persistent or recurrent fever, oxygen desaturation, and radiological progression of bronchopneumonia at the time when the body viral load was expected to fall. ${ }^{7}$ The clinical deterioration was postulated to be mediated by an exaggerated host immunological response to the virus. ${ }^{7}$ This was 
further supported by the observation that systemic corticosteroid treatment at this stage often resulted in clinical and radiological improvement in the patient. Most paediatric patients had relatively mild disease and seldom progressed to phase 3 characterised by acute respiratory distress syndrome and diffuse alveolar damage with or without pulmonary fibrosis. ${ }^{16-18} 20$ It was unclear why children, especially those under the age of 12 years, would be less severely affected. One possible reason related to their prior exposure to other respiratory viruses, making their immune systems more resilient. Others have proposed that young children were not able to mount a "heightened" mature immune response as seen in adult patients during the immune dysregulation phase of SARS and thus less organ damage ensued with its associated morbidity and mortality. Besides, children in general presented with fewer comorbidities than adults. The paediatric patients who developed severe pulmonary disease and required mechanical ventilation came from the adolescent group. ${ }^{16} 20$

\section{HAEMATOLOGICAL, BIOCHEMICAL, AND IMMUNOLOGICAL VARIABLES IN THE ACUTE PHASE OF THE DISEASE}

The most common haematological abnormality was lymphopenia and occurred in $>90 \%$ of patients during the course of illness. ${ }^{16-18} 20$ It was due to destruction of CD4 and CD8 lymphocytes. Other features of low grade disseminated intravascular coagulation, including thrombocytopenia, prolonged activated partial thromboplastin time, and raised Ddimer concentration, were usually found in the more severely affected children. ${ }^{16-18} 20$

Slightly raised lactate dehydrogenase concentration was encountered in $80 \%$ of the paediatric cases. ${ }^{320}$ Other abnormal biochemical indices such as raised serum alanine aminotransferase and creatine kinase concentrations were less often observed. ${ }^{16-18}{ }^{20}$ Circulating bilirubin concentration was not affected. Adolescents with severe clinical signs and symptoms had more deranged biochemical indices, and these variables remained increased for a longer duration. ${ }^{17}$

Chemokines and proinflammatory and anti-inflammatory cytokines were longitudinally monitored in a small group of paediatric patients. ${ }^{21} 22$ Interleukin $1 \beta$, a proinflammatory cytokine, was substantially upregulated, suggesting selective activation of the caspase-l dependent pathway in infected macrophages. ${ }^{21}$ Interferon- $\gamma$-inducible protein-10 (IP-10) and monokine induced by interferon- $\gamma$ (MIG) were also appreciably increased at the acute phase of the infection. ${ }^{22}$ Other important proinflammatory cytokines, interleukin 6 and tumour necrosis factor $\alpha$, were, however, only slightly

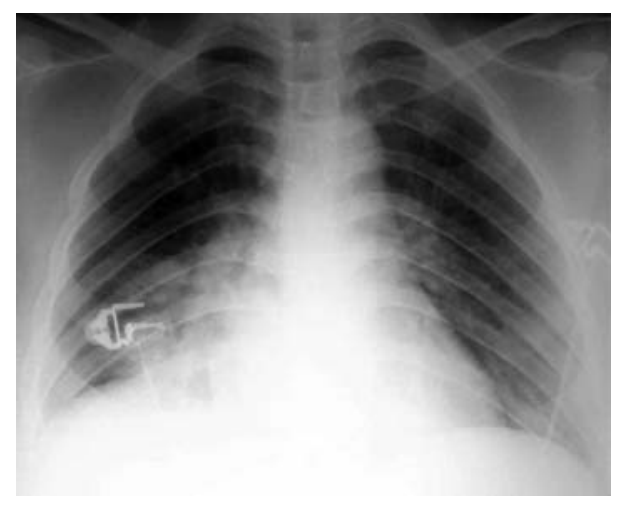

Figure 1 This 12 year old girl with severe acute respiratory syndrome presented with fever, unproductive cough, and constitutional symptoms. The chest radiograph revealed bilateral consolidation of the lower lobes on day 6 after the onset of fever. increased. $^{21}$ In contrast, circulating concentrations of RANTES (regulated upon activation normal $\mathrm{T}$ cell expressed and secreted) remained within the normal range throughout the clinical course. ${ }^{22}$ These results suggest that SARS-CoV predominantly induces type $1 \mathrm{~T}$ helper lymphocyte mediated immune response, which can promote effective viral clearance but may have relatively little influence on immunologically mediated allergic reaction such as hyper-reactive bronchoconstriction. In addition, proteomic analysis of serial plasma samples of paediatric patients with SARS showed the presence of unique signatures, which were increased within the first week of infection, decreased on recovery, and were positively correlated with SARS-CoV viral load. ${ }^{23}$ These results could be used to develop a quick "Protein Chip" assay for detection and monitoring of patients with SARS.

\section{CLINICAL AND RADIOLOGICAL DIAGNOSIS AND RAPID DIAGNOSTIC TEST}

As the presentation of SARS was non-specific and often indistinguishable from other childhood infections, the diagnosis was often difficult unless there was a clear history of contact with an infected patient. The Centers for Disease Control and World Health Organisation had promulgated a case definition for SARS. These definitions and criteria, although applicable to both adults and children, were based mainly on adult experience. ${ }^{24}$ The case definition was useful in guiding clinicians in decision making about treatment. However, as the early symptoms of children affected with SARS were very similar to those of other forms of upper or lower respiratory tract infections, the decision on admission as to whether to isolate and how to treat children presenting with fever but without a definite contact history remained difficult. $^{3}$ Sometimes even the contact history can be misleading. In our hospital, we came across two children who presented with symptoms suggestive of SARS and a definite contact history, but were later diagnosed to have bacterial septicaemia. ${ }^{25}$ The World Health Organisation had subsequently modified the definition of a probable case to include a suspected case of SARS that was positive for SARS coronavirus.

Early chest radiological findings in paediatric patients may be normal or share features of bronchopneumonia common to other respiratory viruses. ${ }^{316-18} 2026$ The characteristic feature of pulmonary SARS-CoV infection was patchy airspace consolidation predominantly located at the periphery of the lungs and in the lower lobes (fig 1). ${ }^{26}$ Other pathologies such as linear atelectasis, peribronchial thickening, ground glass opacities, and focal/multifocal consolidations had been observed, ${ }^{26}$ but radiological features typical of lobar pneumonia or pleural effusion were usually absent. ${ }^{16-18} 20$ High resolution computed tomography (HRCT) scan of the
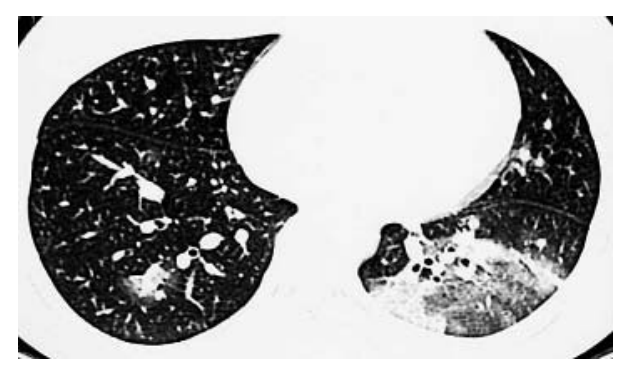

Figure 2 This 14 year old girl with severe acute respiratory syndrome presented with fever, chills, and myalgia. The high resolution computed tomography scan of the thorax on day 4 after the onset of fever showed multifocal areas of mixed ground glass opacification and consolidation in the left lower lobe. There was another smaller area of opacification in the right lower lobe. 
thorax was useful for detecting early airspace disease before the pulmonary lesions were apparent on chest radiographs (fig 2 ). ${ }^{26}$ This investigation with relatively high radiation doses should be reserved for suspected cases with positive contact history and normal chest radiograph findings. Nonetheless, both chest radiograph and HRCT scan could only provide useful information on the severity of pulmonary involvement and the subsequent progression of lung disease. These imaging techniques could not be used as specific tools for the diagnosis of SARS. ${ }^{3}{ }^{16-18} 20$

The diagnostic capability of conventional RT-PCR for detecting SARS-CoV was low during the first week of illness. Its overall sensitivity in nasopharyngeal aspirate specimens and stool samples was estimated to be about 50\% in paediatric patients. ${ }^{20} \mathrm{~A}$ one step, real time RT-PCR has been developed for the quantitative measurement of viral RNA in plasma. ${ }^{27}$ This test detected SARS-CoV RNA in all the cases of paediatric patients studied. ${ }^{27}$ The high sensitivity and quick turnaround time (four to six hours) make it a potentially useful rapid diagnostic tool for clinical practice. Paired acute and convalescent serology for SARS-CoV was useful for confirming the infection (seroconversion or fourfold increase in antibody titres) and for seroepidemiological survey but not in the initial triaging phase of suspected patients. ${ }^{7}$

\section{INFECTION CONTROL, TREATMENTS, AND ADVERSE EFFECTS}

The management of paediatric patients with SARS consists of effective triaging of inpatients, stringent infection control measures, and treatment of SARS-CoV infection. Effective triaging of febrile patients and patients with respiratory symptoms is essential to prevent cross infection among inpatients. ${ }^{28}$ As the initial presenting features of the disease are often non-specific and do not allow an easy and reliable differentiation between SARS and non-SARS cases, the triaging process relies mainly on contact history, presence or absence of fever, and radiological features of bronchopneumonia. Our group has also found that raised serum lactate dehydrogenase concentration in the presence of low neutrophil count and serum creatine phosphokinase concentration at presentation indicated an increased likelihood of SARS infection in young children. ${ }^{3}$ Theoretically, it is best to isolate each suspected case in a negative pressure single room. However, in the absence of such facilities in most acute hospitals, different categories of patients are required to be cohorted into separate groups. ${ }^{28}{ }^{29}$ The ultimate organisation of the cohorting process depends on the geographical configuration of the paediatric ward and neonatal unit. ${ }^{28}$ Parental visiting should be restricted for children with fever, and no visiting should be allowed for suspected and probable cases of SARS. Daily telephone calls and images of children in hospital delivered by electronic mail or real time videophone to parents greatly enhance the communication between patients, family members, and hospital staff.

In principle, all infection control measures are targeted at preventing fomite, droplet, and aerosol spread of the virus. $^{28}{ }^{29}$ Designated "gown-up" and "gown-down" areas should be set up within the high risk areas. A protective outfit, including water resistant gowns, disposable gloves, and particulate respirators (N95 masks), must be worn in these wards. In addition, goggles and face shields are recommended for performing high risk procedures such as collection of potentially contaminated specimens, manipulation of airway or tracheal intubation, and attending delivery of febrile patients. The use of high airflow equipment such as mechanical respirators and suction catheters should ideally be accompanied by an efficient closed circuit scavenger system incorporated with viral filters. However, apparatus without the scavenger system-for example, nebuliser, oxygen mask, and nasal continuous positive airway pressure system - should not be used outside the incubator or in an open ward. ${ }^{28} 29$ Importantly, strict enforcement of hand hygiene is vital to minimise nosocomial spread of the virus.

As the pathogenesis and the underlying immunological response of this newly identified virus have not been fully elucidated, the treatment of SARS-CoV infection has not been standardised in paediatric or adult patients. It is important not to miss any potentially treatable but life threatening bacterial infection or atypical pneumonia. ${ }^{25}$ Children with fever and pneumonia should be covered with broad spectrum antibiotics such as a third generation cephalosporin and a macrolide. In principle, antiviral therapy should be used in the viral replication phase (phase 1), and immunomodulating therapy in the immunopathological phase (phase 2) of the disease. ${ }^{19}$ Ribavirin was empirically prescribed during the outbreak, ${ }^{16-18}{ }^{20}$ but subsequently found to be ineffective against SARS-CoV in an in vitro study. ${ }^{30}$ Systemic corticosteroids may be tried in children with progressive pulmonary disease in the second phase. A close temporal relation has been observed between clinical and radiological improvement and the use of intravenous pulsed methylprednisolone in seriously affected children. ${ }^{18}$ Although anecdotal experience supports the use of systemic corticosteroids in severe cases, this category of drug has been associated with major adverse effects, such as osteonecrosis of long bones, ${ }^{31}{ }^{32}$ and life threatening nosocomial infection. ${ }^{33}$ The current data on cytokines in both paediatric and adult patients with SARS do not support the use of tumour necrosis factor $\alpha$ monoclonal antibody for treatment. ${ }^{2134}$ Lopinavir/ ritonavir $^{35}{ }^{36}$ and integrative Chinese/Western medicine ${ }^{37}$ in subsequent retrospective analysis were found to be associated with clinical improvement, but further randomised placebo controlled trials are needed to substantiate their effects.

\section{OUTCOME AND PROGNOSIS}

Despite the fact that SARS in pregnancy is associated with a high incidence of spontaneous miscarriage, preterm delivery, intrauterine growth retardation, and severe gastrointestinal morbidity, ${ }^{13}{ }^{14}$ there has been no evidence to suggest that the virus is transmitted transplacentally from mother to the newborn. ${ }^{14}$

More importantly, there have been no deaths of young children and adolescents. ${ }^{16-18}{ }^{20}$ It has now been accepted that young children ( $\leqslant 12$ years of age) run a less aggressive clinical course than adolescents and adults. ${ }^{16-18} 20$ It has also been suggested that paediatric patients with severe disease often have more prolonged and deranged haematological and biochemical indices. ${ }^{17}$ Sore throat and peak neutrophil count $>10 \times 10^{9} / 1$ are independent risk factors associated with a severe disease. ${ }^{18}$ Adolescent patients are also more likely to receive pulsed methylprednisolone treatment. ${ }^{18}$

Most children with SARS recovered quickly from the infection..$^{20} \mathrm{~A}$ follow up study on pulmonary function showed that all children were clinically asymptomatic six months after the acute illness. ${ }^{38}$ About $10 \%$ of children had a mild obstructive or restrictive defect on lung function assessment, but substantially more patients (34\%) had abnormal HRCT scan of the thorax. These abnormalities included ground glass opacification $(11 \%)$, air trapping $(17 \%)$, or a combination of these lesions $(6 \%)$. The need for oxygen supplementation and lymphopenia were significant risk factors in predicting these radiological changes. The abnormalities were more prevalent in children with severe disease. ${ }^{38}$ Our group also found that children recovered from SARS have lower peak exercise oxygen consumption than age and sex matched normal controls six months after the disease. The reduction in exercise capacity was significantly greater in the group with persistent HRCT changes than in those whose HRCT had 
shown complete resolution (unpublished data). Other adverse outcomes of SARS and/or side effects of treatment included vague muscle weakness, diffuse hair loss, visual or auditory hallucination, decreased attention span, forgetfulness, mood depression, and emotional liability. ${ }^{20}$ Whether such abnormalities will persist and other sequelae, especially psychobehavioural, have yet to emerge can only be answered by following up these patients and performing comprehensive assessments on a regular basis.

\section{SUMMARY}

- Vertical transmission of SARS-CoV from infected mothers to their newborns has not been observed.

- Young children ( $\leqslant 12$ years) tend to have a milder disease. In contrast, adolescents (13-17 years) have more constitutional features and could run a severe clinical course resembling that of adult patients. No deaths have been reported in paediatric patients affected with SARS.

- A definitive contact history is the most important and reliable predictor of SARS.

- The diagnostic value of conventional RT-PCR assay has been disappointing. One step, real time quantitative RTPCR measurement of SARS-CoV RNA in plasma may potentially be developed as a rapid test for diagnosis of SARS.

- The treatment recommendation is based on our previous experience and modelling of the adult regimen. Randomised placebo controlled trials are needed to formulate standardised treatment for paediatric patients with SARS-CoV infection.

- A proportion of patients have residual radiological changes despite relatively normal lung function and clinical remission. Reduced exercise capacity six months after the acute disease was documented. Longer term follow up is required to assess progression of these abnormalities.

\section{Authors' affiliations}

A M Li, P C Ng, Department of Paediatrics, Prince of Wales Hospital, The Chinese University of Hong Kong

Competing interests: none declared

\section{REFERENCES}

1 Lee N, Hui DS, Wu A, et al. A major outbreak of severe acute respiratory syndrome in Hong Kong. N Engl J Med 2003;348:1986-94.

2 Varia M, Wilson S, Sarwal S, et al. Investigation of a nosocomial outbreak of severe acute respiratory syndrome (SARS) in Toronto, Canada. CMAJ 2003; 169:285-92.

3 Cheng FWT, Ng PC, Chiu WK, et al. A case-control study of SARS versus community acquired pneumonia. Arch Dis Child 2005;90:747-9.

4 Peiris JS, Lai ST, Poon LL, et al. SARS Study Group. Coronavirus as a possible cause of severe acute respiratory syndrome. Lancet 2003;361:1319-25.

5 Drosten C, Gunther S, Preiser W, et al. Identification of a novel coronavirus in patients with severe acute respiratory syndrome. N Engl J Med 2003;348: 1967-76

6 Ksiazek TG, Erdman D, Goldsmith CS, et al. SARS Working Group. A novel coronavirus associated with severe acute respiratory syndrome. N Engl J Med 2003;348: 1953-66

7 Peiris JS, Chu CM, Cheng VC, et al. Clinical progression and viral load in a community outbreak of coronavirus associated-SARS pneumonia: a prospective study. Lancet 2003;361:1767-72.
8 Chan KH, Poon LLM, Cheng VCC, et al. Detection of SARS coronavirus in patients with suspected SARS. Emerg Infect Dis 2004;10:294-9.

9 Kuiken T, Fouchier RA, Schutten M, et al. Newly discovered coronavirus as the primary cause of severe acute respiratory syndrome. Lancet 2003;362:263-70

10 Fouchier RA, Kuiken T, Schutten M, et al. Aetiology: Koch's postulates fulfilled for SARS virus. Nature 2003;423:240.

11 Poutanen SM, Low DE, Henry B, et al. National Microbiology Laboratory Canada; Canadian Severe Acute Respiratory Syndrome Study Team. Identification of severe acute respiratory syndrome in Canada. N Engl J Med 2003;348: 1995-05.

12 Cavanagh D. Nidovirales: a new order comprising Coronaviridae and Arteriviridas. Arch Virol 1997; 142:629-33.

13 Wong SF, Chow KM, Leung TN, et al. Pregnancy and perinatal outcomes of women with severe acute respiratory syndrome. Am J Obstet Gynecol 2004;191:292-7

14 Shek CC, Ng PC, Fung GPG, et al. Infants born to mothers with severe acute respiratory syndrome. Pediatrics 2003;112:e254-6.

15 Ferm VH, Willhite C, Kilham L, et al. Teratogenic effects of ribavirin on hamster and rat embryos. Teratology 1978;17:93-101.

16 Hon KL, Leung CW, Cheng W, et al. Clinical presentations and outcome of severe acute respiratory syndrome in children. Lancet 2003;361:1701-3.

17 Bitnun A, Allen $U$, Heurter $\mathrm{H}$, et al. Children hospitalized with severe acute respiratory syndrome-related illness in Toronto. Pediatrics 2003;112:e261-8.

18 Chiu WK, Cheung PCH, Ng KL, et al. Severe acute respiratory syndrome in children: Experience in a regional hospital in Hong Kong. Pediatr Crit Care Med 2003;4:279-83.

19 Hui DSC, Sung JJY. Severe acute respiratory syndrome. Chest 2003; 124:12-15.

20 Leung CW, Kwan YW, Ko PW, et al. Severe acute respiratory syndrome among children. Pediatrics 2004;113:e535-43.

21 Ng PC, Lam CWK, Li AM, et al. Inflammatory cytokine profile in children with severe acute respiratory syndrome (SARS). Pediatrics 2004;113:e7-14.

$22 \mathrm{Ng}$ PC, Lam CWK, Li AM, et al. Chemokine response in children with SARS. Arch Dis Child 2005;90:422-3.

23 Poon CWP, Chan KCA, Ng PC, et al. Serial analysis of plasma proteomic signatures in pediatric patients with severe acute respiratory syndrome (SARS) and correlation with viral load. Clin Chem 2004;50:1452-5.

24 Centers for Disease Control and Prevention. Clinical guidance on the identification and evaluation of possible SARS-CoV disease among persons presenting with community-acquired illness (version 2) (updated January 8 2004). http://www.cdc.gov/ncidod/sars/clinicalguidance.htm (accessed 12 Oct 2004).

25 Li AM, Hon KLE, Cheng WT, et al. Severe acute respiratory syndrome: "SARS" or "not SARS". J Paediatr Child Health 2004;40:63-5.

26 Babyn PS, Chu WCW, Tsou IYY, et al. Severe acute respiratory syndrome (SARS): chest radiographic features in children. Pediatr Radiol 2004;34:47-58.

$27 \mathrm{Ng}$ EKO, $\mathrm{Ng} \mathrm{PC}$, Hon KLE, et al. Serial analysis of the plasma concentration of SARS-coronavirus RNA in pediatric patients with severe acute respiratory syndrome. Clin Chem 2003;49:2085-8.

28 Leung TF, Ng PC, Cheng FWT, et al. Infection control for SARS in a tertiary paediatric centre in Hong Kong. J Hosp Infect 2004;56:215-22.

$29 \mathrm{Ng} \mathrm{PC}$, So KW, Leung TF, et al. Infection control for SARS in a tertiary neonatal centre. Arch Dis Child 2003;88:F405-9.

30 Health Canada. Management of severe acute respiratory syndrome (SARS) in adults: interim guidance for health care providers. www.hc-sc.gc.ca/pphbdgspsp/sars-sras/pdf/sars-clin-guide-20030703_e.pdf (accessed 10 Oct 2003).

31 Hong N, Du XK. Avascular necrosis of bone in severe acute respiratory syndrome. Clin Radiol 2004;59:602-8.

32 Chan CW, Chiu WK, Chan CC, et al. Osteonecrosis in children with severe acute respiratory syndrome. Pediatr Infect Dis J 2004;23:888-90.

33 Wang HJ, Ding YQ, Li X, et al. Fatal aspergillosis in a patient with SARS who was treated with corticosteroid. N Engl J Med 2003;349:507-8.

34 Wong CK, Lam CWK, Wu AK, et al. Plasma inflammatory cytokines and chemokines in severe acute respiratory syndrome. Clin Exp Immunol 2004; 136:95-103

35 Chan KS, Lai ST, Chu CM, et al. Treatment of severe acute respiratory syndrome with lopinavir/ritonavir: a multicentre retrospective matched cohort study. Hong Kong Med J 2003;9:399-406.

36 Chu CM, Cheng VCC, Hung IFN, et al. Role of lopinavir/ritonavir in the treatment of SARS: initial virological and clinical findings. Thorax 2004;59:252-6.

37 Li J, Li SD, Du N. Clinical study on treatment of severe acute respiratory syndrome with integrative Chinese and Western medicine approach. Zhongguo Zhong Xi Yi Jie He Za Zhi 2004;24:28-31.

38 Li AM, So HK, Chu WCW, et al. Radiological and pulmonary function outcome of children with SARS. Pediatr Pulmonol 2004;38:427-33. 